\title{
Corresponsabilidad familiar y el equilibrio trabajo-familia: medios para mejorar la equidad de género
}

\author{
Verónica Gómez Urrutia \\ Universidad Autónoma de Chile, Talca, Chile. \\ Email: vgomezu@uautonoma.cl \\ Andrés Jiménez Figueroa \\ Universidad de Talca, Talca, Chile. \\ Email: anjimenez@utalca.cl
}

\begin{abstract}
Resumen: Se examina el concepto de corresponsabilidad familiar -que supone la articulación de tareas "productivas” y "reproductivas" desde una perspectiva que armonice los espacios de familia y trabajo de una forma más equitativa entre hombres y mujeres- como un elemento clave para el desarrollo social de los países. Como contribución a su discusión, se revisa aquí los principales antecedentes conceptuales y teóricos dadas las transformaciones sociales, su impacto en las familias y sus equilibrios en distribución de roles y particularmente en la percepción de autoeficacia parental. Se plantea que aunque es un tópico que requiere más investigación, los avances en esta materia sugieren que la corresponsabilidad familiar es un elemento clave para la adquisición de mayores grados de autonomía y sentido de eficacia de los padres, y por ello un factor de importancia para la mejora de las relaciones entre los padres y entre éstos y sus hijos.
\end{abstract}

Palabras clave: Corresponsabilidad, Trabajo-Familia, Autoeficacia Parental, Enfoque de Género.

\section{Family co-responsability and the work-family balance: ways to improve gender equity}

\begin{abstract}
This article examines the concept of family co-responsibility as a key element for social development of countries. Co-responsibility is understood as the articulation of productive and reproductive tasks in a way that it conciliates work and family spaces in a more equitable distribution for men and women, looking for gender equality. In order to contribute to the discussion on this concept, we revise some social transformations, their impact in the family and their equilibrium in the distribution of roles, and particularly in the perspective of their perceived parental self-efficacy. Although more research is needed on this topic, the existing evidence suggests that co-responsibility is crucial to attain higher degrees of personal autonomy and a sense of self-efficacy for parents, and thus a significant importance for the improvement of family relationships, both os the couple as well as of them with their children.

Keywords: Corresponsibility, Work-family balance, parental self-efficacy, gender perspective.
\end{abstract}




\section{Corresponsabilidade familiar e equilíbrio trabalho-família: meios para melhorar a eqüidade de gênero}

Resumo: Examina-se o conceito de corresponsabilidade famíliar -que envolve a articulação de tarefas “produtivas” e "reprodutivas” desde uma perspectiva que harmoniza os espaços de família e do trabalho de uma forma mais equilibrada entre homens e mulheres- como um elemento-chave para o desenvolvimento social dos países. Como contribuição para o debate, nós revemos aqui os principais antecedentes conceituais e teóricos segundo as transformações sociais, o seu impacto sobre as famílias e seus equilibrios na distribuição de papéis e, particularmente, na percepção de auto-eficácia parental. Recomenda-se que embora seja um tema que requer mais pesquisas, o progresso nesta área sugerem que a corresponsabilidade da família é um elemento fundamental para a aquisição de um maior grau de autonomia e senso de eficácia dos pais, e, portanto, um fator importante para melhorar as relações entre os pais e entre estes e os seus filhos.

Palavras-chave: Manejo, Trabalho-Família, Auto-eficácia Parental, Enfoque de gênero.

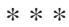

\section{Introducción}

Tradicionalmente, las mujeres han tenido la responsabilidad por el llamado trabajo reproductivo -esto es, el cuidado de la casa y de quienes en ella habitan- como diferenciado del trabajo productivo, que implica intercambios monetarios en el mercado, que estaba reservado a los varones. Algunos cambios sociológicos de importancia, tales como la entrada masiva de las mujeres al mercado de trabajo y la modificación del tamaño de las familias derivada de una caída sostenida en la tasa de fertilidad, han provocado una crisis de este modelo, que ha llevado a replantearse el equilibrio o mejor, la relación- entre dos de los más importantes espacios de la vida de cualquier persona: la familia y el trabajo.

Este proceso de transformación y de reconstrucción de la vida social genera oportunidades para el surgimiento de nuevas formas de vida orientada a nuevas oportunidades, derechos y libertades en la vida familiar y laboral (Brullet, 2010). Tal reconstrucción requiere la reorganización de los roles entre hombres y mujeres, cuyo eje central radica en el compromiso de la pareja en los tiempos destinados en distintos quehaceres en la vida familiar, tales como las tareas domésticas, soporte económico, la educación y el cuidado de los hijos (Torío et al., 2010). El rol de las organizaciones en este ejercicio de redistribución equitativa de los roles en el hogar es importante desde la creación de políticas que permitan la facilitación de la vida laboral y familiar tanto para hombres como para mujeres (Balmforth \& Garden, 2006).

En este contexto, el presente artículo examina el concepto de coresponsabilidad familiar -que supone la articulación de tareas "productivas" y "reproductivas" desde una perspectiva que armonice los espacios de familia y trabajo de una forma más equitativa entre hombres y mujeres- 
como un elemento clave para el desarrollo social de los países. Ello, porque se aborda un aspecto central en la vida de las personas; la familia, definida como un conjunto de individuos unidos por vínculos consanguíneos y de afecto, además es considerada como el núcleo fundamental de la sociedad, cuya función es orientar, responder y satisfacer los requerimientos de sus miembros, vinculándolos con el mundo social (Villarroel y Sánchez, 2002). Cada núcleo familiar tiene una estructura y jerarquía, que condiciona su funcionamiento y la distribución de las tareas que se llevan a cabo dentro del hogar.

\section{Definiciones y teorías en el estudio de la corresponsabilidad familiar}

El término corresponsabilidad familiar ha tenido distintas concepciones que no han sido consensuadas en una definición única. Usualmente, se lo considera como un trabajo no remunerado, entendido como la energía, tiempo y desarrollo de habilidades que resultan determinantes en una familia para llevar a cabo las tareas del hogar y el cuidado de los hijos. El término se ha derivado desde una conceptualización de roles y tareas enmarcado dentro de la sensibilización social que tiene como principal objetivo el lograr la igualdad entre géneros, fomentando de esta forma la conciliación de la vida laboral y familiar (Maganto, Etxeberría \& Porcel, 2010; Rodríguez, Peña, \& Torío, 2010).

En la consecución de dicha conciliación existen elementos relevantes definidos por Maganto, Bartau y Etxeberria (1999, citado en Maganto et al., 2010), quienes han enfatizado en los patrones de comportamiento e interacción que influyen en la distribución y consenso de los roles y tareas repartidas entre los miembros de la familia, definiendo tres características principales de la corresponsabilidad familiar. En primer lugar, se considera la percepción de un reparto justo, entendida como la percepción de los miembros de la familia acerca de la asignación de tareas de manera justa que favorezcan el desarrollo de las personas dentro de la familia y de la familia en sí misma, considerando las capacidades, dificultades de la tarea y el tiempo de cada cual. En segundo lugar, la dimensión actuación coordinada considera la coordinación y establecimiento de las tareas entre los miembros de la familia y modificaciones que se puedan dar en virtud del tiempo y las tareas. En tercer lugar, se considera la dimensión asunción de responsabilidad compartida que implica la realización de tareas, organización y control, de tal modo que la familia como un todo debe responsabilizarse de la dinámica en la realización de las tareas del hogar.

Tal como se aprecia en la definición anterior, la corresponsabilidad familiar mantiene estrecha relación con el trabajo doméstico. Sin embargo, este tipo de trabajo no sólo se orienta a tareas domésticas, sino que también incorpora la crianza de los hijos y su educación (Maganto et al., 2010). Según Shelton y John (1996, citado en Rodríguez, Peña, \& Torío, 2010) 
dichas tareas se agrupan en tres dimensiones principales:

a. Tareas del hogar: Comprende tareas relacionadas con la administración de recursos en el hogar y tareas relacionadas con el aseo y ornato, la alimentación, reparación y transporte en el funcionamiento familiar.

b. Cuidado de los hijos e hijas y de otras personas dependientes: Incluye actividades que van en pos del cuidado, atención y seguridad de estos integrantes de la familia, con acciones orientadas hacia la alimentación, salud, seguridad, acompañamiento, entre otras, que vayan en beneficio de éstas.

c. Trabajo emocional: comprende actividades orientadas al soporte emocional dentro de la familia y que orienta tales actividades a conductas de apoyo, de escucha, aprecio, de amor entre otras conductas que van en beneficio de la dinámica y soporte emocional dentro de los integrantes del hogar.

De ello se desprende que la participación debe ser congruente con las responsabilidades de cada integrante de la familia. Así, diversos estudios (Spitze, 1991; Thomson \& Walter, 1991; Mereder, 1993, citados en Maganto, Etxeberría \& Porcel, 2010) destacan la presencia de dos roles principales en las tareas intrafamiliares. Por un lado, se encuentra el rol del ejecutor encargado principalmente de la realización de las tareas domésticas como lavar, cocinar, planchar, entre otras. Por otro lado, consideran el rol del organizador el cual destaca por un rol más invisible, cuya tarea principal radica en asegurarse y planificar las acciones de la familia, el tomar decisiones y asignar tareas. Generalmente, la invisibilidad del rol del organizador se encuentra representado por la mujer enmarcada en el ámbito familiar, cuyo rol muchas veces es ignorado.

Vosler (1996, citado en Maganto, Bartau y Etxeberria, 2003) ha sido más específico en la distinción de roles dentro del trabajo familiar, señalando roles o tareas básicas en la dinámica de la corresponsabilidad familiar o trabajo familiar, de los cuales se pueden definir:

a. Rol del proveedor: Las tareas de este rol se orientan a la provisión de recursos básicos para la supervivencia de la familia, como es el caso de elementos de higiene, alimentación, salud, vestimenta, entre otras.

b. Rol de liderazgo familiar y de toma de decisiones: Considerando a la unidad familiar como un grupo, se espera de quien ejerce este rol, mantener una función de desarrollar patrones comunicacionales entre los miembros de la familia y también con personas ajenas a esta unidad, relacionado también con aspectos como el poder compartido y el control de comportamientos. 
c. Rol de mantenimiento y organización del hogar: las tareas de este rol se orientan en una gran variedad de ejercicios, tal es el caso de realizar las compras del hogar, preparar las comidas del día, la limpieza y un sinfín de actividades repetitivas que suelen estar acompañadas de mucho tiempo y energía.

d. Rol del cuidador de los miembros dependientes: la persona que cumple con este rol debe asumir el cuidado de las personas en la familia que requieren de cierta dependencia, tal es el caso de los niños, ancianos o miembros con algún tipo de discapacidad, brindando el cuidado y la atención necesaria a éstos.

e. Rol del educador y supervisor: el rol de esta persona se orienta a la supervisión de niños y adolescentes tanto en temas educativos, de crianza y socialización, por medio de asignación de tareas que incluyen estimulación afectiva y cognitiva.

\section{Corresponsabilidad familiar y distribución de roles}

A la luz de la clasificación anterior, podemos preguntarnos -considerando los datos disponibles- si los cambios en la familia chilena incluyen una redistribución de responsabilidades dentro de la familia. Como observan Olavarría (2004) y Valdés (2007c), uno de los puntos más difíciles de implementar, dentro de la agenda de igualdad de género, ha sido la incorporación de los varones a las tareas domésticas y de cuidado. Para Olavarría, la creencia- generalizada desde mediados de los 90- de que los varones en los últimos años participan cada vez más en las actividades domésticas y de crianza, en una relación de mayor igualdad con la mujer y de cercanía con los hijos, es una hipótesis relativamente frágil, que lleva a que la familia se declare de una forma (mayor igualdad) y se construya de otra, más apegada a los patrones tradiciones.

Algunos datos pueden ayudarnos a situar esta afirmación. Los pocos estudios sobre la materia sugieren que hay interés en los varones jóvenes por ser participantes activos en la crianza de sus hijos: el estudio de Aguayo, Correa y Cristi (2011), basado en una encuesta representativa para Santiago, Valparaíso y Concepción, reporta que ocho de cada diez hombres entre 18 y 59 años que tienen hijos con los que viven y están en el empleo remunerado $(75,9 \%)$ indicaron que "me gustaría trabajar menos si eso significara pasar más tiempo con mis hijos”, mientras un $61,7 \%$ señaló que “dedican muy poco tiempo a sus hijos por motivos de trabajo”. Sin embargo, en el mismo estudio, 61,9\% señaló estar de acuerdo con la afirmación: “Mi rol en el cuidado de los niños es principalmente como ayudante”. Solamente el $7,7 \%$ de los varones indica proveer cuidado a sus hijos diariamente, situación relacionada con la inserción laboral de ellos y sus parejas (Aguayo, Correa y Cristi 2011:59) ${ }^{1}$, y 46\% de los hombres está total o parcialmente de acuerdo con la afirmación: “Cambiar pañales, bañar y alimentar a los niños o niñas es responsabilidad de la madre”. Este mismo estudio propone un 
índice de cuidado para estimar cuánto se involucran los varones en el cuidado de sus hijos, el cual resultó ser bastante bajo, particularmente en lo que se refiere a actividades como cuidar de niños enfermos, cocinar y lavar para ellos. No se encontraron diferencias significativas por edad, $(\mathrm{F}=0,690$; $p=0,558$ ), mientras que con el nivel educacional la asociación estuvo en los límites de los niveles de confianza establecidos al 95\% ( $F=2,927 ; p=0,6)$ (Aguayo, Correa y Cristi 2011:62).

En una línea similar, la encuesta sobre la Primera Infancia realizada por la JUNJI en 2010 en una muestra representativa a nivel nacional de menores de 0 a 5 años 11 meses y sus progenitores, residentes en zonas urbanas, mostró que en el $74 \%$ de los casos el cuidado principal estaba a cargo de la madre, especialmente entre los 0 y los 2 años, seguido del cuidado proporcionado por otro pariente con un 21\% (ENPI 2010:60) habitualmente, otra mujer (Valdés et al, 2006). Tal vez más interesante, esta encuesta sugiere que el tipo de actividad que hombres y mujeres realizan con los hijos e hijas es también diferenciada: los roles de mantenimiento y organización doméstica del hogar, así como del cuidado de dependientes (particularmente lo referido a la reproducción cotidiana de los cuerpos) siguen siendo predominante femeninos, mientras que el rol del educador y supervisor (que incluye supervisar las tareas escolares e imponer disciplina) tiende a una mayor equidad entre ambos padres. Ambos estudios son concordantes con los datos aportados por el estudio del PNUD para Chile (2010), en el cual se señala que, independientemente de su situación ocupacional, los hombres tienen un bajo índice de involucramiento en las tareas del hogar, mientras que las mujeres -trabajen fuera del hogar o no- mantienen niveles similares de trabajo en el hogar. Referente a este tópico las esposas, suelen informar emociones negativas hacia el trabajo doméstico incluyendo estrés, frustración cansancio y aburrimiento (Gager, 1998 en Kawamura y Brow, 2010). Sin embargo estos sentimientos negativos hacia el trabajo del hogar no siempre se traducen en percepciones de injusticia en la división de las tareas, lo cual revela una discrepancia importante entre el comportamiento individual y la dinámica emocional (Kawamura y Brow, 2010).

Es en este contexto que surge el concepto de sobrecarga de roles, el cual hace referencia a las situaciones en que los roles de una persona en la familia llevan consigo responsabilidades que sobrepasan las capacidades propias de la persona para sobrellevarlas. Se ha demostrado que las personas más susceptibles a padecer de esta sobrecarga son las mujeres trabajadoras, y es allí donde surge la importancia de las organizaciones en entregar el apoyo adecuado a las personas que forman parte de ésta, de forma que exista una corresponsabilidad entre las empresas y las familias (Maganto, Bartau \& Etxeberria, 2003). Vosler (1996, citado en Maganto, Etxeberría \& Porcel, 2010) señala que el prestar ayuda a las familias en la identificación, planificación y organización de sus recursos, puede otorgarles una estrategia adecuada para sobrellevar la sobrecarga de roles.

También se ha observado que existe una tendencia considerable hacia una opinión positiva sobre el reparto justo en las tareas domésticas, 
pero que en la ejecución de estas opiniones en conductas concretas de corresponsabilidad familiar, distan considerablemente, ya que el cambio se ha observado en las actitudes de las personas pero no en sus conductas de manera significativa (Olavarría 2004; Rodríguez, Peña, y Torío, 2010). Gran parte de los hombres que deciden insertarse en el trabajo del hogar, muestran conductas con intenciones de cooperar con las tareas que ejecuta la mujer. Sin embargo, esta intención de cooperación o colaboración aún se encuentra distante a un reparto equitativo, manifestándose una congruencia entre intención y conducta -con una mayor prevalencia-, en jóvenes y en hombres con parejas con ejercicio laboral fuera del hogar (Torío, Rodríguez, Fernández, \& Molina, 2010). Olavarría señala que los hombres declaran querer involucrarse, en alguna medida, en la crianza de los hijos cualquiera sea su condición social y edad; sin embargo, en la reproducción doméstica de la vida cotidiana -que incluye actividades como bañar, alimentar, cocinar para niños- "se sienten extraños y consideran que es un ámbito ajeno, salvo en aquellas actividades que reafirman la masculinidad dominante" (2004: 217) como los arreglos de la vivienda, los trámites fuera del hogar y algunos aspectos del presupuesto familiar. Sobre este mismo punto Bartau, Etxebarria y Maganto (2003) señalan que la participación masculina en las tareas domésticas ha cambiado paulatinamente, logrando una mayor participación en aquellas que tradicionalmente son consideradas femeninas, sin embargo, sigue siendo la mujer la que invierte más tiempo en las tareas domésticas, a pesar de tener una jornada laboral completa (INE, 2008).

En el mundo contemporáneo existe una creencia social marcada, aunque con ciertas modificaciones en el último tiempo, en virtud de que el hombre es quien debe hacerse cargo de las labores de soporte económico de la familia y trabajar fuera del hogar, mientras que a la mujer se le considera con labores más orientadas al cuidado del hogar y de los hijos, lo cual determina los patrones de comportamiento familiar (Maganto, Etxeberría \& Porcel, 2010). La visión del "varón proveedor” y la "mujer cuidadora” permanecen en la familia chilena, aunque con importantes matices según clase social y edad (Olavarría, 2004; PNUD, 2010; Valdés, 2006). Con dichos matices, actualmente se han modificado ciertas prácticas relacionadas con modelos de tipo patriarcal imperantes en el pasado; en este ámbito, cambios importantes se han realizado principalmente en materia de legalidades, que pueden alterar relaciones de poder, siempre orientadas hacia la apertura de oportunidades que permitan la construcción de vínculos familiares más democráticos (Brullet, 2010).

El aprovechamiento de estas oportunidades dependerá, desde luego, de las posibilidades que las personas tengan de negociar sus roles y responsabilidades familiares, tanto en el hogar como en el mundo del trabajo. Dentro de las variables involucradas en la distribución de roles entre hombres y mujeres en la familia, se observa que el poder dentro de la pareja se encuentra fuertemente influenciado por los aspectos económicos. Así, la teoría de los recursos argumenta que el cónyuge que posee más recursos, es quien tiene más poder sobre la familia de forma que mantiene menos implicación en el trabajo familiar (Rodríguez, Peña \& Torío, 2010). Las expli- 
caciones argumentadas bajo la teoría de género estiman que las diferencias se dan a nivel de actitudes hacia la corresponsabilidad familiar según la cultura imperante ${ }^{2}$ : el trabajo doméstico, sea remunerado o no, sintetiza la reflexión en torno a la división sexual del trabajo entre lo productivo y lo reproductivo, a la naturalización de la valoración diferenciada de lo público y lo privado y, finalmente a la asignación de los papeles de género desiguales para hombres y mujeres en la sociedad (Consejo Nacional para Prevenir la Discriminación, 2008); asignación que incluye la distribución de bienes materiales y simbólicos, como la contribución social presentada por el trabajo remunerado y el no remunerado. Por su parte, la teoría de disponibilidad temporal argumenta que las personas que invierten mayor cantidad de tiempo en el trabajo productivo, dedican menos tiempo a las tareas domésticas en el hogar (Rodríguez, Peña \& Torío, 2010) -aunque los datos disponibles para el caso chileno muestran que lo contrario no es necesariamente verdadero (PNUD, 2010).

Desde el punto de vista de los recursos, la incorporación de la mujer al mercado laboral ha ido más rápido que la adopción de mayores responsabilidades de los varones en el trabajo no remunerado, afectando la disponibilidad de tiempo de las mujeres. Así, hoy en día las mujeres se han incorporado al sistema laboral, aportando económicamente al hogar y realizando una doble jornada, ya que continúan siendo responsables de la mayor parte del trabajo doméstico, aún cuando están empleadas y pasan la mayor parte de su tiempo fuera del hogar. Esta sobrecarga de responsabilidades que asume la mujer ya sea en el trabajo doméstico o en el remunerado, además de la dificultad para compatibilizar la vida profesional y la familiar, puede tener efectos negativos, dañando su salud y en algunos casos afectando su desarrollo familiar y laboral (Artázcoz, Borrell, Rohlfs, Beni, Moncada, Benach, 2001).

El Instituto Nacional de Estadísticas (2008) por medio de la aplicación de encuestas sobre el uso del tiempo, devela la inequidad de género existente en la distribución de roles al interior de los hogares. Dicha asimetría queda reflejada al observar la carga de trabajo de hombres y mujeres: las mujeres, además de tener un empleo remunerado, siguen teniendo bajo su responsabilidad la mayor proporción de las tareas del hogar, las que incluyen actividades tales como la preparación de alimentos, hacer el aseo, lavar la ropa, administrar el hogar, pagar cuentas, etc.

Por ejemplo, en relación a las tareas domésticas realizadas de lunes a viernes, se observa que cerca de ocho de cada diez mujeres realizan por lo menos media hora este tipo de actividades, contra menos de cuatro de cada diez hombres. En el caso del cuidado de miembros del hogar, sólo 9,2\% de los hombres dedica tiempo para ello, a diferencia del 35,9\% de las mujeres. El predominio femenino en las actividades relacionadas con trabajo doméstico no remunerado es evidente, incluso si sólo se consideran los fines de semana. Si bien hay un notable aumento de la participación masculina (del $37,7 \%$ al 57,3\% de la población de doce años y más), en lo que respecta a la mujeres, ésta incluso aumenta a un $81,9 \%$ de las personas. Otros estudios 
sugieren que en el caso de las mujeres casadas y con independencia económica, son ellas quienes realizan la mayor parte de la mano de obra familiar, gastando un promedio de 19 horas por semana a las tareas domésticas en comparación con los hombres quienes sólo dedican 10 horas a la semana (Kawamura y Brow, 2010).

De acuerdo a Rodríguez, Peña y Torió (2010) la distribución del trabajo familiar en función del género se entiende como un fenómeno socialmente construido y estructuralmente constreñido y que durante los años se ha ido modificando, en donde el hombre se ha visto más involucrado, sin embargo, aún existen diferencias en función del género, ya que si bien la experiencia del trabajo doméstico ya no es ajena a los hombres, la fuerza de los convencionalismos de género todavía se deja sentir dentro y fuera del hogar. Parte de estos convencionalismos se refleja, también, en la asimetría salarial que todavía existe en nuestro país entre hombres y mujeres, y que se refleja en brechas de ingreso por sexo de hasta 35\% (INE 2010: 11 Encuesta suplementaria de ingreso), particularmente en el segmento con mayor educación -que, en el caso de las mujeres, es el que tiene mayor participación en el mercado de trabajo (CASEN 2011) ${ }^{3}$. Desde el punto de vista de los recursos económicos, las mujeres ganan menos que los varones, aun controlando por número de horas trabajadas, nivel educacional y tipo de ocupación (INE 2010).

Asimismo, el estudio realizado por Rodríguez, Peña y Torió (2010) indica que los acuerdos sobre el reparto del trabajo doméstico son soluciones flexibles y prácticas que permiten resolver problemas que se presentan en el día a día, en los que no se invierte mucho tiempo dialogando acerca de cómo ha de ser este reparto. Por ello, no suele haber procesos de negociación en los que se usen reglas y procedimientos explícitos y elaborados.

Tanto hombres como mujeres realizan tareas domésticas, pero la forma en que éstas se negocian refleja la persistencia del dualismo de género. Esto se debe a la fuerza de los patrones tradicionales, ya que el trabajo doméstico tiene un significado totalmente distinto para cada persona y que en muchas ocasiones condicionaría la negociación sobre el reparto. De este modo, las discusiones y los conflictos que se generan por la diferente visión de este tema, se puede resumir en que para las mujeres las responsabilidades domésticas son prioritarias, mientras que los varones, aunque participen, son conscientes, y lo asumen, de que desempeñan un papel secundario. A pesar de esto, los hombres argumentan que son las mujeres a las que les cuesta delegar y que son muy exigentes en cuanto al nivel de desempeño doméstico. Sin embargo, ellas expresan su disconformidad por la escasa participación de sus parejas (Rodríguez, Peña y Torió, 2010; Olavarría, 2004).

\section{Corresponsabilidad y autoeficacia parental}

La distribución de tareas dentro del hogar y su conciliación (o falta de) con la vida laboral puede resultar una fuente de tensión familiar, particu- 
larmente para quienes son responsables de funcionamiento doméstico y financiero del hogar. Sin duda dentro de la familia, uno de los subsistemas que en mayor medida debe afrontar numerosos eventos estresantes es el constituido por ambos progenitores. Su función supone poner en juego múltiples habilidades o competencias para cubrir las necesidades de los hijos, y hacerlo de una manera competente (Rodrigo, Martín, Cabrera y Máiquez, 2009 en Pérez, Lorence, Menéndez, 2010).

Las competencias o habilidades hacen referencia a la capacidad que poseen las personas para generar y coordinar respuestas (afecto, cognición, comunicación y comportamiento) flexibles y adaptativas a corto y a largo plazo ante las demandas asociadas a la realización de sus tareas vitales y generar estrategias para aprovechar las oportunidades que les brindan los contextos de desarrollo (Waters y Sroufe, 1983 en Rodrigo, Máiquez, Martín y Byrne, 2008). Por otra parte, el sistema de creencias y expectativas ante ciertas actividades o áreas de la vida, determina cómo las personas evalúan su desempeño como padres -en otras palabras, su sentido de autoeficacia parental.

Para contextualizar mejor este concepto y su aplicación al problema que nos ocupa, permítasenos situar la noción de autoeficacia. De acuerdo a la teoría de Bandura (1993), existen dos tipos de expectativas relacionadas con la eficacia. La primera de ellas corresponde a la autoeficacia o eficacia personal; la cual hace referencia al juicio que el propio sujeto realiza de sí mismo ante su capacidad para alcanzar ciertos resultados. La segunda expectativa es la de resultados, entendida como las consecuencias que el sujeto espera que ocurran ante su intervención. Si ambas expectativas se mueven hacia el mismo sentido, y se caracterizan por poseer un sentido positivo, la conducta se ve reforzada, y es probable que se actúe nuevamente de la misma forma.

La percepción de la autoeficacia, además, se refiere a las creencias que poseen las personas acerca de sus propias capacidades para lograr determinados resultados (Farkas-Klein, 2008). Estos resultados serán variables, debido a que las personas no pueden realizar todas las actividades bien, ya que eso requeriría poseer un dominio total de cada aspecto de la vida. Estas creencias de sus propias capacidades para conseguir el logro difieren entre áreas determinadas, es decir, el sentido de eficacia y los niveles de ésta, no será el mismo en todas las actividades que realice o en cada aspecto de la vida (Bandura, 2006).

De acuerdo a Bandura (2006), el sistema de creencias no es un rasgo global, más bien es un grupo de autocreencias ligadas a ámbitos de funcionamiento diferenciado, por este motivo no todas las actividades que realiza una persona posee el mismo sentido o nivel de eficacia. Este sentido de eficacia personal es el fundamento de la acción humana, que se ve afectada cuando las personas creen que para producir efectos deseados tienen pocos incentivos para actuar o para perseverar ante las dificultades (Bandura, 1999). Además, desempeña un papel fundamental en el funcionamiento hu- 
mano, debido a que afecta el comportamiento de las personas, pero no sólo directamente, sino también por medio de su impacto en otros determinantes claves, tales como metas y aspiraciones, expectativas de resultados, tendencias afectivas, percepción de los impedimentos y oportunidades que se presenten en el medio social que rodee a los individuos (Farkas-Klein, 2008).

Las creencias de eficacia influyen en los pensamientos de las personas, tanto auto-estimulantes como auto-desvalorizantes, optimismo o pesimismo; los cuales llevarían a tomar determinados cursos de acción, planteándose desafíos y metas, comprometiéndose con los mismos; además, estas creencias determinarán la cantidad de esfuerzo que se invierte en determinados emprendimientos, esperando ciertos resultados producto de sus esfuerzos. Muchas de estas acciones deberán enfrentar ciertos obstáculos para poder cumplirse, y será la autoeficacia la que mediará la magnitud de su perseverancia ante estos obstáculos, resistiendo de este modo a la adversidad. Se lidiará además con niveles de estrés y depresión que experimentan cuando se enfrentan con demandas exigentes del ambiente y los logros que alcanzan (Bandura, 2006).

El sentimiento de autoeficacia al ser entendido como una evaluación que realiza el propio individuo ante su propia capacidad para conseguir lo que desea, influye directamente en el grado de implicancia y persistencia que se llevará a cabo en tareas difíciles y podría afectar en la resolución de dichas tareas (Reina, Oliva y Parra, 2010). En términos del problema que nos ocupa, esta evaluación -y las expectativas sobre las que se basa- pueden verse tensionadas en la medida en que, como observa Ximena Valdés (2007c), las formas tradicionales de ser padre y madre se desdibujan, en un escenario donde hay una demanda por una mayor participación de los hombres en la esfera doméstica, mientras las mujeres asumen nuevos roles -incluyendo el de proveedora y/o jefa de hogar en números crecientes (CASEN 2011). Así, "Nuevas formas de ejercer la parentalidad por parte de hombres y mujeres se instalan en las generaciones jóvenes, cuyos comportamientos tienden a diluir las viejas concepciones de maternidad y paternidad” (Valdés 2007c: 3).

Las habilidades que deben emplear los padres, corresponden a las competencias parentales, que según Rodrigo et. al. (2008) son un conjunto de capacidades que permiten a los padres afrontar de modo flexible y adaptativo la tarea de ser padres, considerando las necesidades evolutivas y educativas de sus hijos, de acuerdo a lo considerado aceptable por la sociedad. De este modo la valoración que cada padre y madre hace de sus habilidades parentales parece ser más importante de lo que parece, ya que la percepción positiva del desempeño como padre o madre propicia respuestas más adaptativas ante situaciones complejas, repercutiendo en mejores niveles de satisfacción parental (Pérez, Lorence y Menéndez, 2010).

Las habilidades que los padres perciben de sí mismos, que los hacen sentirse padres competentes, están sobre la base de sus propias opiniones y experiencias. Esta Autoeficacia Parental, es considerada como la capaci- 
dad para realizar de manera competente y eficaz una tarea concreta, conllevando a experiencias de éxito o fracaso, generando un aumento o disminución de la autoeficacia (Kendall y Bloomfield, 2005). El ser padres es una tarea bastante compleja y la percepción que posea cada uno de ellos varía de acuerdo a las funciones que desarrollen dentro de su vínculo familiar. De acuerdo a esto, y en relación a estudios realizados, existe evidencia de la influencia del género y de la edad en la percepción del papel de los padres. En este sentido las madres enfatizan con mayor frecuencia el apego, la sensibilidad y responsabilidad como características de su rol, esto en contraste con lo que hacen los padres (Turiano, 2001 en Farkas-Klein, 2008).

Culturalmente, quien ocupa el rol de estar a cargo del cuidado de los hijos es la madre, mientras que el padre posee el rol de proveedor. A partir de esto, diversos autores concuerdan que transcurridos los primeros años de vida del bebé, las funciones de los padres difieren entre sí, conllevando a que el rol del cuidado del bebé posea connotaciones diferentes en padres y madres $^{4}$. Esto ha generado que el estudio de la eficacia personal considere dominios distintos en ambos (Farkas-Klein, 2008).

Por su parte la eficacia parental se refiere a las creencias personales sobre las propias capacidades para lograr ser un "buen padre". En este sentido, los padres que creen en sus propias habilidades se sentirán generalmente más satisfechos y capaces de hacer lo necesario para perseverar y lograr una tarea determinada, ello incluye tener expectativas reales y ser capaces de percibirse a sí mismos como padres competentes (Kendall y Bloomfield, 2005 en Farkas, 2008).

Sobre la base de lo señalado es que se hace relevante destacar la importancia que tienen los dominios del trabajo y la familia en la vida de las personas, tomando en cuenta que cuando se es madre o padre, con todas las exigencias y responsabilidades que esto implica, la tarea de equilibrar estos dos dominios se complejiza aún más. Estudios muestran que la angustia emocional que produce la dificultad de compatibilizar las demandas y responsabilidades del trabajo y la familia se relacionan con problemas entre padres e hijos y con conductas negativas en éstos (Crouter et. al., 1999 en Lau, 2009; Valk y Srinivasan, 2011).

Por otra parte, existe evidencia que señala que hombres y mujeres no compatibilizan las demandas de ambos dominios de la misma forma, esto en cuanto al tiempo, nivel de compromiso y equilibrio en la satisfacción. Componentes que contribuyen a lograr un ajuste y equilibrio entre el trabajo y la familia (Greenhaus, Collins y Shaw, 2003). Pese a lo anterior, Aguirre y Martínez (2006) afirman que no existe diferencia en los niveles de conflicto experimentados por ambos sexos, argumentando que esto se debe a un cambio en el modelo de pareja, lo que implicaría una instancia en donde ambos desarrollan una carrera profesional y comparten sus responsabilidades familiares.

Ahora bien, independiente del género, estudios argumentan que las personas que experimentan estrés en el trabajo tienen interacciones hosti- 
les dentro del núcleo familiar, respondiendo con más sanciones a los comportamientos de sus hijos. Además la investigación sugiere que aquellos que están físicamente y emocionalmente fatigados por el trabajo tienden a ser menos sensibles, participativos y solidarios con sus hijos. Ello tiene directa relación con el término de Autoeficacia Parental, entendida como un factor importante en la elección de las conductas que los padres utilizan en la crianza de sus hijos, los esfuerzos que realizan para adaptarse a las diversas situaciones que se presentan y la perseverancia que muestran ante los obstáculos (Almeida, 1999 en Lim y Leng, 2003; Bandura, 1977; Repetti y Wood, 1997 en Lim y Leng, 2003).

En este sentido, el conflicto que se puede dar al tratar de conciliar el trabajo y familia presenta una correlación significativa y negativa con la calidad percibida en la interacción con los hijos y con la Autoeficacia Parental. Es decir, los niveles más altos de conflicto trabajo- familia están asociados a percepciones más bajas en la calidad de la interacción con los hijos y en la autoeficacia de los padres. Por tanto, al tener altos niveles de conflicto entre ambos dominios, se vería afectada la calidad de la interacción entre padres e hijos, y el sentimiento de autoeficacia que los primeros experimentan (Gali, Weisel y Tzuk, 2007; Lau, 2009).

En síntesis, las experiencias vividas en un dominio tenderían a afectar en el otro, y en consecuencia en la autoeficacia de los padres y la interacción que éstos tienen con sus hijos. Por lo tanto, en este contexto hay que señalar que si los padres creen en sus propias habilidades se sentirán más satisfechos y capaces de hacer lo necesario para perseverar y lograr una tarea determinada, lo que traería consigo tener expectativas reales y además, ser capaces de percibirse a sí mismos como padres competentes (Farkas-Klein, 2008). Desde esta perspectiva, la corresponsabilidad familiar resulta crucial tanto para la adquisición de mayores grados de autonomía y sentido de eficacia de los padres, como para propender a una mayor calidad de interacción entre padres, madres ${ }^{5}$ e hijos.

La valoración que cada padre y madre hace de sus habilidades parentales propicia respuestas más adaptativas ante situaciones complejas, repercutiendo en mejores niveles de satisfacción parental (Pérez, Lorence, Menéndez, 2010). Es decir, quienes evalúan positivamente su rol parental, son más capaces de actuar anticipadamente antes las necesidades de sus hijos. En este contexto, se hace relevante investigar la autoeficacia parental y su relación con una redistribución de los roles familiares y parentales tradicionales. Ello, debido a que los cambios ocurridos en la estructura familiar han generado que los padres pasen menos tiempo en el hogar, y por ende la interacción con sus hijos es menos frecuente y asumirá formas diversificadas en la medida en que hombres y mujeres adopten nuevas formas de organizar sus responsabilidades familiares y laborales. De este modo, si los padres lograr conciliar sus roles satisfactoriamente y se evalúan positivamente tenderán a esforzarse por satisfacer las necesidades de sus hijos, a pesar del poco tiempo que tienen y de las diversas responsabilidades que implican el trabajo y la familia (Reina, Oliva y Parra, 2010). 


\section{Conclusiones}

Históricamente, los roles dentro de la familia han estado diferenciados por el sexo que posea cada individuo y por las pautas entregadas por la cultura en la cual están insertos. Esta visión social de lo que debe hacer una persona, determina el papel que ejerce dentro de su núcleo familiar. En el caso del rol de la mujer, éste ha estado basado en los quehaceres del hogar y en la crianza y cuidado de los hijos, centrando toda su atención a la mantención de éste y la preocupación por cada integrante de su familia. Por el contrario, el rol de los hombres, ha estado relacionado con el mantenimiento económico de la familia, enfatizando su rol de proveedor. Sin embargo, esta visión cultural respecto a los roles de los hombres y las mujeres, se ha ido transformando en la medida en que los hombres y particularmente las mujeres han asumido roles en el espacio público, generando cambios radicales en las dinámicas familiares y en el sector económico y laboral (Cifre y Salanova, 2004).

La distribución desigual del trabajo dentro de la casa es a menudo justificada por las expectativas sociales en donde se pone a los hombres como sostén de la familia y a las mujeres como amas de casa. En este sentido, el menor trabajo que ejerce el marido en las labores del hogar se justifica por la demanda de su rol de proveedor, es decir que trabaja fuera del hogar y contribuye con ingresos a éste -una imagen normativa de la familia que representa cada vez menos a la población chilena, donde el 39\% de los hogares tienen como cabeza a una mujer y, dentro de los hogares monoparentales ${ }^{6}$, los hogares con jefatura femenina son una amplia mayoría (CASEN 2011). Este cambio en el modo de organización familiar, junto con la incorporación de la mujer al mercado laboral, daría cuenta de una modificación crucial en el funcionamiento en los hogares, debido al aumento de responsabilidades para la mujer, implicando un cambio a nivel laboral y familiar según Arriagada (2005, en Jiménez, González y Reyes, 2009). Así, si bien los hombres han comenzado a participar más en los quehaceres del hogar y en el cuidado de los hijos (Rodríguez, Peña y Torió, 2010), las investigaciones existentes sugieren que los hombres, en comparación con las mujeres, se muestran poco dispuestos a organizar su tiempo libre para colaborar con los quehaceres de sus parejas (Aguayo, Correa y Cristi, 2011; Cifre y Salanova, 2004; Olavarría, 2004; Thompson, 1991 en Kawamura y Brow, 2010;).

A partir de lo anterior, toma relevancia el tema del equilibrio entre la vida laboral y familiar, ya que al interactuar ambos mundos se influyen positiva o negativamente, generando equilibrio o conflicto. Con la inclusión de la mujer al sistema laboral han ocurrido cambios en la dinámica familiar y en la distribución de las tareas domésticas, como por ejemplo el tiempo que ambos dedican a estar físicamente en el hogar y las actividades que desarrollan dentro de éste (Rodríguez, Peña y Torió, 2010). Por tanto, ante las demandas de estas esferas, lo esperable sería que cada uno cumpliera con las exigencias de ambos mundos sin descuidar el otro, desarrollándose de manera satisfactoria en los dos. Es decir, que puedan mantener 
las relaciones familiares que se consideren deseables y al mismo tiempo conseguir un nivel de desarrollo profesional satisfactorio (OIT, 2009; Papí, 2005).

Lograr este equilibrio -esto es, no sólo conciliación, sino conciliación con co-responsabilidad7 implicará, necesariamente, una redistribución de la tareas dentro y fuera del hogar: nuevas exigencia en ambos entornos requieren que las parejas vayan adecuando su funcionamiento a las nuevas responsabilidades que asumen (Cordón, Corrales y Nicolás, 2010; Valdés, 2009). Esto es necesario, como hemos argumentado, por al menos tres razones: a) equidad de género, para reducir las brechas de recursos (tiempo, dinero e incluso los riesgos de salud asociados a una doble jornada) que hoy existen entre hombres y mujeres y que ayudan a la perpetuación de patrones tradicionales por sexo; b) la demanda por una mayor armonía entre las que quizás sean las esferas más importantes en la vida de las personas, y c) la necesidad de dar respuesta social a la cuestión del cuidado de los hijos y otros dependientes, de manera que la relación entre trabajo y familia no sea una de suma-cero (esto es, una relación donde más dedicación a una esfera significa, necesariamente, menoscabo para la otra). Hasta ahora, como observa Inés Reca (2008), las estrategias desde el sector público y privado han favorecido sólo la conciliación, sin que necesariamente se busque impactar en la distribución de tareas dentro del hogar. Ello puede resultar contraproducente para una mejora en las relaciones familiares -a menudo vistas como en crisis- desde el punto de vista de la presión que enfrentan los progenitores y la reproducción de desigualdades sociales y de género para una próxima generación.

Esto porque, como hemos argumentado a lo largo de estas páginas, la tarea educativa que los padres realizan es muy compleja, ya que deben promover el desarrollo de los menores. Para esto utilizan un conjunto de capacidades que les permiten afrontar de modo flexible y adaptativo la tarea de ser padres, considerando las necesidades evolutivas y educativas de sus hijos, de acuerdo a lo considerado aceptable por la sociedad - identificadas como competencias o habilidades parentales (Rodrigo et al., 2008). Aunque es un tópico que requiere más investigación, los avances en esta materia sugieren que la corresponsabilidad familiar es un elemento clave para la adquisición de mayores grados de autonomía y sentido de eficacia de los padres -lo que en este artículo hemos denominado autoeficacia parental- , y por ello un factor de importancia para la mejora de las relaciones entre los padres y entre éstos y sus hijos. Como apuntan (Maganto, Etxeberría \& Porcel, 2010), las interacciones en el medio familiar (incluida la distribución de responsabilidades en casa) producen el proceso en el cual se adquieren los principios básicos de convivencia social, el cual tiene repercusiones considerables en el mundo social-laboral de la persona y de la futura familia. Goodnow (1996, citado en Maganto, Bartau \& Etxeberria, 2003), explica la importancia de otorgarle al niño responsabilidades en virtud de la corresponsabilidad en su familia, esperando una transición entre un pensamiento individualista en la cual sus preocupaciones se basan en sus propias responsabilidades y necesidades, a una visión más cercana a la responsabilidad familiar. 


\section{Notas}

${ }^{1}$ No se encontraron relaciones ni con la edad $(\mathrm{p}=0,979)$ ni con el nivel educacional $(\mathrm{p}=0,158)$. Sin embargo, los autores reportan que, cuando ambos miembros de la pareja trabajan, $49,1 \%$ de los hombres señaló que cuidaban a sus hijos de manera compartida con su pareja, mientras que un 46,8\% declaró que lo hacía siempre o usualmente la mujer; cuando sólo el varón trabaja, un 86,2\% de los hombres señaló que la pareja cuidaba siempre o usualmente a los hijos/as, apenas un 12,1\% lo hacía de manera compartida y un $1,7 \%$ siempre o usualmente él (frente a $4,1 \%$ en el caso anterior). Sin embargo, entre los hombres que no tenían un empleo remunerado, "no se aprecia la misma tendencia anterior, ya que las mujeres declararon en mayor proporción cuidar a los hijos/as respecto a sus parejas hombres desempleados. Situación similar cuando ninguno de ellos trabaja, donde la mayoría de los hombres $(62,1 \%)$ indicó que la pareja cuidaba más a los hijos” (2011:60), relación que sí es significativa.

${ }^{2}$ Por medio de la construcción social y cultural, se han otorgado significados y valores diferentes al hecho de ser hombre o mujer, lo cual ha estado basado en ideales respecto del papel y capacidades de cada uno de ellos, considerándose generalmente inferior el rol de la mujer. A partir de esta idea se plantea que las tareas domésticas sean consideradas tareas naturales y propias de las mujeres, teniendo un valor social menor al desarrollado en la esfera pública y productiva definida como masculina (Consejo Nacional para Prevenir la Discriminación, 2008).

${ }^{3}$ Según la encuesta CASEN, en 2011 en el segmento de mujeres con educación superior completa $76,8 \%$ trabaja fuera del hogar, mientras que en el segmento con educación media completa lo hace el 55,6\% (37,8\% en el segmento de educación superior incompleta).

${ }^{4}$ El concepto de familia en nuestro país ha ido cambiando paulatinamente; entre muchas otras cosas por el aumento de los matrimonios con contrayentes que tienen edades más tardías y más años de educación formal. Ambos fenómenos han ocasionado que los niños ingresen precozmente a algún tipo de cuidado institucional o permanezcan con sus abuelos u otros parientes. Ello contribuye a que la familia comparta sus roles clásicos y la función educativa de los menores (Vargas, 2001).

${ }^{5}$ Tradicionalmente las mujeres han sido socializadas para asumir la responsabilidad de cuidar a los hijos y realizar las tareas dentro de la casa. Lo que genera que éstas tiendan a interactuar más y compartir más íntimamente con sus hijos (Galinsky, 1999 en Lim y Leng, 2003).

${ }^{6}$ Se entiende por hogar monoparental el constituido por jefe(a) de hogar sin cónyuge o conviviente, con hijos o hijastros de cualquier estado civil. Se incluyen los hogares extensos y compuestos.

${ }^{7}$ Como hemos señalado anteriormente, entendemos por corresponsabilidad parental, a la cantidad de energía, tiempo y el desarrollo de las habilidades necesarias para atender las tareas propias del hogar y el cuidado de los hijos/as, cuya finalidad es el mantenimiento armónico del sistema familiar (Bartau, Etxebarria y Maganto, 2003). 


\section{Bibliografía}

Aguayo, F., Correa, P., \& Cristi, P. (2011). Encuesta IMAGES Chile. Resultados de la encuesta internacional de masculinidades y equidad de género. CulturaSalud/EME. Santiago.

Aguirre, Z. y Martínez, M. P. (2006). “Influencia de la situación laboral en el ajuste familia-trabajo”. Mapfre Medicina 17(1), 14-24.

Artázcoz, L., Borrell, C., Rohlfs, I., Beni, C., Moncada, A. y Benach, J. (2001). “Trabajo doméstico, género y salud en población ocupada”. Gac Sanit, 15(2), 150-153.

Balmforth, K. \& Garden, D. (2006). “Conflict and facilitation between work and family: realizing the outcomes for organizations”. New Zealand Journal of Psychology, 35(2), 69-76.

Bandura, A. (1977). "Self-efficacy: toward a unifying theory of behavioral change”. Psychological Review, 84(2), 191-215.

Bandura, A. (1993). "Perceived self- efficacy in cognitive development and functioning”. Educ. Psychol, 28(2), 117- 148.

Bandura, A. (1999). "Self-Efficacy Pathways to Childhood Depression”. Journal of Personality and Social Psychology, 76(2), 258-269.

Bandura, A. (2006). Self-efficacy beliefs of adolescents. Recuperado el día 26 de Mayo del 2011 desde http://des.emory.edu/mfp/014BanduraGuide2006.pdf

Brullet, C. (2010). “Cambios familiares y nuevas políticas sociales en España y Cataluña. El cuidado de la vida cotidiana a lo largo del ciclo de vida”. Educar, 45, 51-79.

Cifre, E. y Salanova, M. (2004). “Estrategias de conciliación familia/trabajo: buscando la calidad de vida”. Estudios Financieros, 259, 11- 154.

Cordón, M., Corrales, R. y Nicolás, I. (2010). Material de corresponsabilidad en el ámbito familiar. Instituto Andaluz de la Mujer. Recuperado el día 12 de Junio del 2011 desde http://www.educarenigualdad.org/media/pdf/uploaded/ old/Mat_208_AMPA_corresp.pdf

ENPI 2010 Encuesta Nacional de la Primera Infancia, ENPI 2010 - Resultados Preliminares. JUNI/UNESCO/Unicef, Santiago.

Farkas-Klein, C. (2008). “Escala de Evaluación Parental (EEP): desarrollo, propiedades psicométricas y aplicaciones”. Universitas Psychologica, 7(2), 457-467. 
Polis, Revista Latinoamericana, Volumen 14, $N^{\circ}$ 40, 2015

Greenhaus, J., Collins, K. y Shaw, J. (2003). “The relation between workfamily balance and quality of life”. Journal of Vocational Behavior, 63, 510531.

INE (2010) Nueva Encuesta Suplementaria de Ingresos (NESI). Recuperado el día 27 de Septiembre 2013 desde http://estudios.sernam.cl/documentos/?eMjA1NTYwMw=--Nueva_Encuesta_Suplementaria_ de_Ingresos_\%28NESI\%29

Instituto Nacional de Estadísticas (2008). Encuesta experimental sobre uso del tiempo en el gran Santiago. Recuperado el 21 de abril del 2010 desde http://www.ine.cl/canales/sala_prensa/noticias/2008/mayo/pdf/ presentacion300508.pdf

Instituto Nacional de Estadísticas (2012). Informe de empleo regional: Región del Maule. Recuperado el día 29 de agosto 2012 desde http:// www.inemaule.cl/archivos/files/pdf/Empleo/2011/julio-aeptiembre/ jul_sep11.pdf

JUNJI/UNESCO/Unicef (2010) Encuesta Nacional de la Primera Infancia, ENPI 2010 - Resultados Preliminares. JUNI/UNESCO/Unicef, Santiago.

Kawamura, S. y Brown, S. (2010). “Mattering and wives’ perceived fairness of the division of household labor”. Social Science Research, 39, 976-986.

Kendall, S. y Bloomfield, L. (2005). "Developing and validating a tool to measure parenting self-efficacy”. Journal of Advanced Nursing, 51(2), 174181.

Lau, Y. (2009). “The impact of Fathers’ work and family conflicts on children's self- esteem: the Hong Kong case”. Social Indicators Research, 95, 363376.

Lim, V. y Leng, G. (2003). “Effects of parental job insecurity and parenting behaviors on youths self-efficacy and work attitudes”. Journal of Vocational Behavior, 63(1), 86-98.

Maganto, J.M., Bartau, I. y Etxeberria, J. (2003). “La participación en el trabajo familiar: Un reto educativo y social”. Revista Electrónica de Investigación y Evaluación Educativa, 9(2), 160-183.

Maganto, J.M., Etxeberria, J. y Porcel, A. (2010). “La corresponsabilidad entre los miembros de la familia, como factor de conciliación”. Educatio Siglo XXI, 28(1), 69-84.

Ministerio Desarrollo Social (2011) Mujer y Familia. Resultados CASEN 2011. Recuperado el día 27 de Septiembre 2013 desde http:// centrodelafamilia.uc.cl/index.php?option=com_content $\&$ view=section \&id=37\&Itemid=591. 
OIT (2009). Trabajo y familia: Hacia nuevas formas de conciliación con corresponsabilidad social. OIT/ PNUD, Santiago.

Olavarría, J. (2004) “¿Dónde está el nuevo padre? De la retórica a la práctica”, en Valdés X. \& Valdés T. (Eds.), Familia y vida privada: ¿transformaciones, resistencias o nuevos sentidos?, pp. 215-250. CEDEM/FLACSO, Santiago.

Organización Internacional del Trabajo y Programa de las Naciones Unidas para el Desarrollo (2009). Trabajo y familia: hacia nuevas formas de conciliación con corresponsabilidad social. Recuperado el día 27 de abril 2012 desde http://www.pnud.cl/areas/GobernabilidadDemocratica/2010/ Informe\%20trabajo\%20y\%20familia/informecompleto_esp.pdf

Papí, N. (2005). “La conciliación de la vida laboral y familiar como proyecto de calidad de vida desde la igualdad”, Reis 5, 91-107.

Pérez, J., Lorence, B., Menéndez, S. (2010). “Estrés y competencia parental: un estudio con madres y padres trabajadores”. Suma Psicológica, 17(1), 47-57.

Programa de las Naciones Unidas para el Desarrollo (PNUD) (2010) Género: los desafíos de la igualdad. PNUD, Santiago de Chile.

Reca, I. (2008) Políticas para la conciliación de las responsabilidades laborales y familiares en Argentina, Chile, Paraguay y Uruguay. Documento de consultoría. Santiago: Programa de la Naciones Unidas para el Desarrollo (PNUD)/ Organización Internacional del Trabajo (OIT).

Reina, M., Oliva, A. y Parra, Á. (2010). "Percepciones de autoevaluación: Autoestima, atoeficacia y satisfacción vital en la adolescencia”. Psychology, Society \& Education. 2(1), 47-59.

Rodrigo, M., Máiquez, M. L., Martín, J. C. y Byrne, S. (2008). Preservación Familiar: un enfoque positivo para la intervención con familias. Pirámide, Madrid.

Rodríguez, M.C., Peña, J.V. y Torío, S. (2010). “Corresponsabilidad familiar: negociación e intercambio en la división del trabajo doméstico”. Papers, 95(1), 95-117.

Torío, S., Peña, J.C., Rodríguez, M.C., Fernandez, C.M y Molina, S. (2010). "Hacia la corresponsabilidad familiar: Construir lo cotidiano. Un programa de educación parental”. EducatioSiglo XXI, 28(1), 85-108.

Valdés et al (2006) ¿“Modelos familiares emergentes o fractura del modelo tradicional?” en: Valdés X., Castelain-Meunier C. y Palacios M. (eds.), Puertas adentro. Masculino y femenino en la sociedad contemporánea, pp. 54-102. Santiago. LOM. 
Valdés, X. (2007b). La vida en común. Transformaciones familiares en Chile y el medio rural en la segunda mitad del siglo XX. LOM, Santiago.

Ídem (2007a). Notas sobre la metamorfosis de la familia en Chile. Documento preparado para la reunión de especialistas "Futuro de las familias y desafíos para las políticas públicas”, CEPAL-UNFPA, realizada en Santiago, de 22 a 23 de noviembre.

Ídem (2007b). Lo privado y lo público: lugares de desigual disputa. Documento preparado para la mesa pro-género de la Fundación Chile 21. Recuperado el día 23 de septiembre 2013 desde http://www.lazoblanco.org/wpcontent/uploads/2013/08manual/bibliog/material_masculinidades_0308.pdf

Ídem (2009) “El lugar que habita el padre en Chile contemporáneo. Estudio de las representaciones sobre la paternidad en distintos grupos sociales”. Polis, Revista de la Universidad Bolivariana, Volumen 8, N²3, pp. 385410.

Vargas, N. (2001). “Cambios en la familia: repercusiones en la práctica pediátrica”. Rev. chil. Pediatr, 72(2), 77-80.

Villaroel, G. y Sánchez, X. (2002). “Relación familia y escuela: un estudio comparativo en la ruralidad”. Estudios Pedagógicos. 28, 123-141.

Recibido: 08.10.2013

Aceptado: 04.06.2014 